\title{
Serum Fetuin-A Evaluation in Chronic Hepatitis C-Virus Patients with Concomitant Type 2 Diabetes Mellitus
}

\author{
Abeer M. Nafee ${ }^{1}$, Noha E. Shaheen ${ }^{1}$, Heba F. Pasha ${ }^{2}$,Mai Saad ${ }^{1}$ \\ ${ }^{1}$ Tropical Medicine Department, Faculty of Medicine, Zagazig University, Egypt \\ ${ }^{2}$ Medical Biochemistry Department, Faculty of Medicine, Zagazig University, Egypt
}

Corresponding Author Abeer M. Nafee

Mobile: 00201005623987

E mail: abeer-n2009 @hotmail.com

Key words: $H C V$ Fetuin-A diabetes
Background and study aim: Hepatitis C virus infection represents a potential public health problem worldwide. Many studies have shown markedly higher prevalence of insulin resistance (IR) and type 2 diabetes mellitus (T2DM) among patients with hepatitis $\mathrm{C}$ virus (HCV) infection compared to healthy subjects. Fetuin-A is one of the principal hepatokines regulating the human metabolism. This study aimed to estimate the level of Fetuin-A in HCV patients with concomitant T2DM, and its relation to insulin resistance.

Patients and Methods: The study was conducted on 115 subjects, 87 patients had chronic HCV infection with and without T2DM, diabetic patients without $\mathrm{HCV}$ infection and 28 healthy control, they were subjected to full history taking thorough clinical examination, abdominal ultrasonography, routine laboratory investigation including fasting and post prandial blood glucose level and assessment of HOMA- IR, viral markers and serum Fetuin-A level by ELISA.

Results: There was high statistically significant increase of serum Fetuin-A level among HCV with DM patient group

\section{INTRODUCTION}

Infection of hepatitis $\mathrm{C}$ virus (HCV) is the main cause of viral hepatitis that may cause chronic hepatitis in about $60-80 \%$ of patients and also can progress to liver fibrosis, liver cirrhosis and hepatocellular carcinoma at the end [1]. Also, hepatitis $C$ virus infection has many extrahepatic manifestations [2].Chronic hepatitis $C$ virus infection is associated with metabolic disorders like increased risk of hyperglycemia and even type-2 diabetes mellitus $[\mathbf{3 , 4}]$.

Glucose intolerance is more common in $\mathrm{HCV}$ related chronic hepatitis than any other cause of chronic liver diseases followed by $\mathrm{HCV}$ group when compared to DM and control groups. Serum Fetuin-A level was higher in $\mathrm{HCV}$ patients than non HCV subjects with statistically significant difference.Also,it was higher among diabetic patients than non diabetic subjects with statistically significant difference.Serum Fetuin-A level was positively correlated with glucose homeostasis parameters in both HCV and HCV\&D.M patients with high statistically significant difference. At Cut-off value of serum Fetuin-A $>5.2 \mathrm{mg} / \mathrm{mL}$, Sensitivity was 54.84 , Specificity was 96.30 , positive PV 94.4 and negative PV was 65.0 for prediction of IR in HCV patients. While, at Cut-off value of serum Fetuin-A >4.9 $\mathrm{mg} / \mathrm{mL}$, sensitivity was 72.41 , specificity was 79.31, positive PV was 77.8 and negative PV was 74.2 for prediction of $\mathrm{DM}$ in HCV patients.

Conclusion : Fetuin-A may have role as a mediator in IR of either HCV or diabetes. However, Fetuin-A is more specific for evaluation of IR than DM in HCV patients as other factors may be incriminated in the pathogenesis of DM in $\mathrm{HCV}$ patients other than serum Fetuin-A level alone.

such as hepatitis B virus infection [5]. Hepatitis $C$ virus infection is associated with insulin resistance in the main pathways of gluconeogenesis and glycolysis. So, chronic HCV infection may predispose the host directly to glucose metabolism abnormalities and act as a risk factor for the development of type 2 diabetes [6,7].

A link between chronic HCV infection and metabolic disorders such as noninsulin-dependent diabetes mellitus (NIDDM) has been suggested. Several studies from various parts of the world found that between $13 \%$ and $33 \%$ of 
patients with chronic hepatitis $\mathrm{C}$ (CHC) have diabetis mellitus (DM). Insulin resistance (IR) is also a common feature of disturbed carbohydrate metabolism in liver disease with or without cirrhosis and precedes the development of DM in these patients [8]. Many results showed that hepatitis $\mathrm{C}$ virus infection can lead to the increased prevalence of type 2 diabetes mellitus [9].

Several mechanisms control the pathogenesis of insulin resistance and T2DM caused by HCV. Those mechanisms could be either direct effect of the virus on insulin signaling [10] or indirect effect via inflammatory cytokines induction such as tumor necrosis factor- alpha (TNF- $\alpha$ ) [11]. The role of cytokines and adipokines in insulin resistance caused by $\mathrm{HCV}$ has been investigated by many studies, but the role of hepatokines in those patients still understudied [12].

HCV-related insulin resistance, glucose metabolic changes and diabetes have important clinical outcomes, such as rapidly progressive fibrogenesis, higher incidence of hepatocellular carcinoma and also reduction of the virological response to interferon- $\alpha$-based therapy [13]. Insulin resistance is a critical component of type 2 diabetes mellitus pathogenesis. Several mechanisms are likely to be involved in the pathogenesis of HCV-related insulin resistance [14]. Impairment of insulin receptor substrate (IRS)-1 and insulin receptor substrate (IRS)-2 expression has been observed in the liver of patients with chronic HCV infection, as well as in $\mathrm{HCV}$ core transgenic mice, $\mathrm{HCV}$ mediates dysfunction of the insulin signaling pathways via several distinct mechanisms, such as upregulating the expression of suppressors of cytokine signaling 3 expression [15], down regulation of peroxisome proliferator-activated receptors gamma (PPAR $\gamma$ ), activation of mammalian target of rapamycin pathway, and increased tumor necrosis factor- $\alpha$ (TNF- $\alpha)$ secretion [16].

Hepatokines are liver-derived proteins that affect the lipid and glucose metabolism in the liver, adipose tissue and skeletal muscle. The dysregulated hepatokines have been considered as risk factors for the development of insulin resistance and type 2 diabetes [17]. Among the principal hepatokines regulating the human metabolism is the Human alpha-2 heremansschmid glycoprotein (known also as Fetuin-A) [18].

Serum Fetuin-A is a multifunctional glycoprotein that is mainly secreted from hepatocytes in human [19]. Fetuin-A has an important role in the protection from vascular calcification by solubilization of calcium and phosphorus in serum ${ }^{(20)}$.Also, Fetuin-A can inhibit the activity of insulin receptor tyrosine kinase by blocking the autophosphorylation of insulin receptor substrate- 1 and tyrosine kinase, and induce a low grade inflammation that will result in insulin resistance [17] and its comorbidities as metabolic syndrome and also type 2 diabetes [21]. So, this work aimed to estimate the level of Fetuin-A in HCV patients with concomitant T2DM, and its relation to insulin resistance.

\section{PATIENTS AND METHODS}

This case-control study included 115 subjects aged (39-57) years, selected from Tropical Medicine Department, Faculty of Medicine, Zagazig University Hospitals. Eighty seven patients had chronic HCV infection with and without T2DM, diabetic patients (T2DM)) without $\mathrm{HCV}$ infection and 28 healthy controls. Diagnosis of HCV chronic hepatitis was based on elevated serum alanine aminotransferase more than 2 times the upper limit of normal level in at least two assessments in prior six months, standard serological assay and liver biopsy (or fibroscan if available) [22].

Diagnosis of diabetes was based on the World Health Organization, as a single raised glucose reading with symptoms, otherwise raised values on two occasions, of either fasting plasma glucose $\geq 7.0 \mathrm{mmol} / \mathrm{l}(126 \mathrm{mg} / \mathrm{dl})$, or with a glucose tolerance test, two hours after the oral dose, the plasma glucose $\geq 11.1 \mathrm{mmol} / \mathrm{l}(200 \mathrm{mg} / \mathrm{dl})$ and glycosylated hemoglobin $\geq 6.5 \%$ [23].

Informed consents were obtained from all patients; this study was approved by Ethical committee of faculty of medicine, Zagazig University, in the period from April 2016 to May 2017. The studied individuals were age and sex matched, randomly selected and divided into 4 groups :

- Group I: Chronic HCV patients without diabetes (29 subjects).

- Group II: Chronic HCV patients with diabetes (29 subjects).

- Group III: Diabetic patients (T2DM) without HCV (29 subjects).

- Group IV: Healthy controls (28 subjects).

\section{Exclusion Criteria :}

- Cirrhotic patients. 
- Patients with renal impairment.

- Patients with malignancies including HCC.

- Patients with chronic hepatitis other than HCV (HBV and autoimmune hepatitis).

- Patients with body mass index $>30$.

- Drugs affecting lipid profile.

All participants were subjected to the following:

- Complete history taking thorough clinical examination .Body mass index (BMI) [24].

- Abdominal Ultrasound was done using sonoscape C11.

- Biochemical assessments, including :

- Complete Blood Count (using Sysmex-xs500i).

- Liver function tests including: bilirubin level, serum albumin, ALT, AST (using Cobas 6000).

- Coagulation profile including: prothrombin time, INR (Sysmex CA-1500).

- Kidney function tests including: serum creatinine and serum urea (using Cobas 6000).

- Virological markers: HCV antibody, HBVsAg, HBcIg Musing (Cobas é 411). HVC RNA was done by PCR. LKMA, ASMA and ANA.

- Alfa feto protein using ELISA kits.

- Fasting blood glucose, postprandial blood glucose and glycosylated hemoglobin .Fasting serum insulin. (Normal adult level 0.7-9 $\mu \mathrm{lU} / \mathrm{ml}$ ).Insulin resistance determined by the homeostasis model of assessment (HOMA-IR) using the formula of: [fasting blood glucose $(\mathrm{mg} / \mathrm{dL}) \times$ fasting insulin (IU/mL)]/405.Normal range 0.5-1.4, above 1.9 indicates early insulin resistance and above 2.9 indicates significant insulin resistance [25].

- Lipid profile: Total cholesterol (TC), LDL cholesterol, HDL cholesterol and triglycerides.

- Evaluation of Fetuin-A level determined by commercially available Human Fetuin-A (FETU-A) ELISA kit from Bioneovanco, Ltd.

- Liver biopsy for HCV patients (or fibroscan if available).

\section{Statistical Analysis :}

The collected data were computerized and statistically analyzed using SPSS program (Statistical Package for Social Science) version 20. Data were tested for normal distribution using the Kolmogorov-Smirnov test. Qualitative data were represented as frequencies and relative percentages. Chi square test $(\chi 2)$ and Fisher exact was used to calculate difference between qualitative variables as indicated. Quantitative data were expressed as mean \pm SD (Standard deviation).Independent $T$ test was used to calculate difference between quantitative variables in two groups in normally distributed data. Oneway ANOVA F-test was used to calculate difference between quantitative variables in more than two groups in normally distributed data. Pearson correlation coefficient used to calculate correlation between two quantitative variables. All statistical comparisons were two tailed with significance level of $\mathrm{P}$-value $\leq 0.05$ indicates significant, $\mathrm{P}<0.001$ indicates highly significant difference while, $\mathrm{P}>0.05$ indicates Non-significant difference. The receiver operator characteristic (ROC) curve was constructed to permit selection of threshold values for test results and comparison of different testing strategies. Areas under ROC curves and their standard errors were determined using the method of Centor, and compared using the normal distribution, with correction for correlation of observations derived from the same cases. A larger area under a ROC curve (AUC) indicates superior test performance, with 1 representing $100 \%$ sensitivity and specificity and 0.5 representing no discriminatory utility.

\section{RESULTS}

There is statistically significant difference as regard BMI among studied groups with higher value reported in $\mathrm{HCV} \& \mathrm{DM}$ and DM patients (Table 1). There was statistically significant difference of ALT, AST levels among studied groups which were higher in HCV \& DM followed by HCV when compared to DM and control groups (Data not shown). There was statistically significant difference as regard lipid profile among different groups, where LDL, TG, TC levels were elevated significantly and HDL level was decreased in HCV\&DM followed by DM when compared to $\mathrm{HCV}$ and control groups (Data not shown).

There is high statistically significant increase of glucose homeostasis parameters among HCV \& DM patients group when compared to other groups (Table 2).

As regard FBS, 2-hour postprandial blood glucose and HbA1C there are high statistically significant differences among different group except HCV vs. control. According to insulin level, there is statistically significant difference 
among different groups. Moreover, HOMA-IR reveals statistically significant difference among all groups except HCV\&DM vs. DM (Table 3).

There is high statistically significant increase of serum Fetuin-A level among HCV \& DM followed by HCV patient groups when compared to DM and control groups (Table 4).

Serum Fetuin-A level is higher in HCV patients than non $\mathrm{HCV}$ subjects with statistically significant difference. Also, it is higher among diabetic patients than non diabetic subjects with statistically significant difference (Table 6).

There was a highly significant positive correlation between Fetuin-A and BMI among all studied subjects (Data not shown). Also, there was positive correlation between serum Fetuin-A and TG, LDL and negative correlation with HDL level with significant difference in the whole studied subjects (Data not shown).
Serum Fetuin-A level is positively correlated with glucose homeostasis parameters in both HCV and HCV\&D.M patients with high statistically significant difference. Moreover, serum Fetuin-A level is inversely correlated with age in $\mathrm{HCV}$ patients with statistically significant difference. Also, negative correlations between serum Fetuin-A and liver enzymes were reported among each group but without statistically significant difference (Table 7).

At Cut-off value of serum Fetuin-A $>5.2 \mathrm{mg} / \mathrm{mL}$, Sensitivity 54.84, Specificity 96.30 , positive PV 94.4 and negative PV 65.0 for prediction of IR in $\mathrm{HCV}$ patients (Table 8, Figure 1).

At Cut-off value of serum Fetuin-A $>4.9 \mathrm{mg} / \mathrm{mL}$, sensitivity was 72.41 , specificity was 79.31 , positive PV was 77.8 and negative PV was 74.2 for prediction of DM in HCV patients (Table 9, Figure 2).

Table (1): Demographic data among studied groups.

\begin{tabular}{|c|c|c|c|c|c|c|c|}
\hline & \multicolumn{4}{|c|}{ Groups } & \multirow{2}{*}{$\begin{array}{c}\text { Total } \\
\text { N=115 }\end{array}$} & \multirow[b]{2}{*}{$\mathbf{p}$} \\
\hline & & $\begin{array}{l}\mathrm{HCV} \\
\mathrm{N}=29\end{array}$ & $\begin{array}{c}\text { HCV\&DM } \\
\quad \mathrm{N}=29\end{array}$ & $\begin{array}{c}\text { DM } \\
\mathrm{N}=29\end{array}$ & $\begin{array}{c}\text { Control } \\
\mathrm{N}=28\end{array}$ & & \\
\hline \multirow[t]{2}{*}{ Sex } & Female & $15(51.7 \%)$ & $15(51.7 \%)$ & $14(48.3 \%)$ & $14(50.0 \%)$ & $58(50.4 \%)$ & \multirow{2}{*}{$\begin{array}{c}0.992 \\
(\mathrm{NS}\end{array}$} \\
\hline & Male & $14(48.3 \%)$ & $14(48.3 \%)$ & $15(51.7 \%)$ & $14(50.0 \%)$ & $57(49.6 \%)$ & \\
\hline \multicolumn{2}{|c|}{ Age, years } & $46.4 \pm 3.4$ & $46.6 \pm 3.4$ & $47.5 \pm 4$ & $44.8 \pm 3.5$ & $46.3 \pm 3.7$ & $\begin{array}{c}0.062 \\
\text { (NS) }\end{array}$ \\
\hline \multicolumn{2}{|c|}{ BMI, $\mathrm{Kg} / \mathrm{m}^{2}$} & $22.3 \pm 1.6$ & $24.4 \pm 1.7$ & $24.4 \pm 1.6$ & $22.2 \pm 1.6$ & $23.3 \pm 2$ & $\begin{array}{c}<0.001 \\
(\mathrm{HS})\end{array}$ \\
\hline
\end{tabular}

HS: Highly Significant, S: Significant, NS:Non Significant.

Table (2): Glucose homeostasis parameters among studied groups.

\begin{tabular}{|c|c|c|c|c|c|c|}
\hline & \multicolumn{4}{|c|}{ Groups } & \multirow{2}{*}{$\begin{array}{c}\text { Total } \\
\mathbf{N}=115\end{array}$} & \multirow[b]{2}{*}{$\mathbf{P}$} \\
\hline & $\begin{array}{l}\mathrm{HCV} \\
\mathrm{N}=29\end{array}$ & $\begin{array}{c}\text { HCV\&DM } \\
\text { N=29 }\end{array}$ & $\begin{array}{c}\mathrm{DM} \\
\mathrm{N}=\mathbf{2 9}\end{array}$ & $\begin{array}{c}\text { Control } \\
\mathrm{N}=28\end{array}$ & & \\
\hline $\begin{array}{l}\text { FBS, mg/Dl } \\
(\text { Mean } \pm \text { SD) }\end{array}$ & $85.3 \pm 16.9$ & $118.2 \pm 31.4$ & $154.9 \pm 28.2$ & $81.1 \pm 11.6$ & $110.1 \pm 37.8$ & $\begin{array}{c}<0.001 \\
\text { (HS) }\end{array}$ \\
\hline $\begin{array}{l}\text { 2-hour } \\
\text { postprandial bl. } \\
\text { glucose,mg/dl } \\
\text { (Mean } \pm \text { SD) }\end{array}$ & $187.6 \pm 14.7$ & $256.8 \pm 29.4$ & $234.7 \pm 24.3$ & $137.3 \pm 9.4$ & $211.6 \pm 35.3$ & $\begin{array}{c}<0.001 \\
(\mathrm{HS})\end{array}$ \\
\hline $\begin{array}{l}\text { HbA1C, g\% } \\
(\text { Mean } \pm \text { SD) }\end{array}$ & $5.5 \pm 0.4$ & $6.8 \pm 1.3$ & $8.2 \pm 0.9$ & $5.1 \pm 0.2$ & $6.4 \pm 1.5$ & $\begin{array}{c}<0.001 \\
(\mathrm{HS}) \\
\end{array}$ \\
\hline $\begin{array}{l}\text { Insulin, IU/mL } \\
(\text { Mean } \pm \text { SD) }\end{array}$ & $8 \pm 0.5$ & $8.6 \pm 0.9$ & $7.6 \pm 0.9$ & $5 \pm 0.7$ & $7.3 \pm 1.6$ & $\begin{array}{c}<0.001 \\
\text { (HS) }\end{array}$ \\
\hline $\begin{array}{l}\text { HOMA-IR } \\
(\text { Mean } \pm \text { SD) }\end{array}$ & $1.6 \pm 0.4$ & $2.9 \pm 0.8$ & $2.5 \pm 0.9$ & $1 \pm 0.2$ & $3.8 \pm 18.9$ & $\begin{array}{c}<0.001 \\
(\mathrm{HS})\end{array}$ \\
\hline
\end{tabular}

HS: Highly Significant, S: Significant, NS: Non Significant.

N.B: Normal level of FBS from 70 to $100 \mathrm{mg} / \mathrm{dL}$, HbA1C 4.8-5.9\%, Insulin 0.7-9 $\mu \mathrm{lU} / \mathrm{ml}$ and HOMA-IR 0.5-1.4. 
Table (3): Glucose homeostasis parameters among different groups (LSD Post-hoc test).

\begin{tabular}{|c|c|c|c|c|c|c|}
\hline & $\begin{array}{c}\text { HCV\&DM } \\
\text { vs. DM } \\
\end{array}$ & $\begin{array}{l}\text { HCV\&DM } \\
\text { vs. Control }\end{array}$ & $\begin{array}{l}\text { DM vs. } \\
\text { Control }\end{array}$ & $\begin{array}{c}\text { HCV\&DM } \\
\text { vs. HCV }\end{array}$ & $\begin{array}{c}\text { DM vs. } \\
\text { HCV }\end{array}$ & $\begin{array}{l}\text { HCV vs } \\
\text { Control }\end{array}$ \\
\hline FBS, $\mathrm{mg} / \mathrm{dL}$ & $\begin{array}{c}<0.001 \\
(\mathrm{HS})\end{array}$ & $\begin{array}{c}<0.001 \\
(\mathrm{HS})\end{array}$ & $\begin{array}{c}<0.001 \\
(\mathrm{HS})\end{array}$ & $\begin{array}{c}<0.001 \\
(\mathrm{HS})\end{array}$ & $\begin{array}{l}<0.001 \\
(\mathrm{HS})\end{array}$ & $\begin{array}{c}0.502 \\
(\mathrm{NS})\end{array}$ \\
\hline $\begin{array}{l}\text { 2-hour } \\
\text { postprandial bl. } \\
\text { glucose, } \mathrm{mg} / \mathrm{dl}\end{array}$ & $\begin{array}{c}<0.001 \\
\text { (HS) }\end{array}$ & $\begin{array}{c}<0.001 \\
(\mathrm{HS})\end{array}$ & $\begin{array}{l}<0.001 \\
(\mathrm{HS})\end{array}$ & $\begin{array}{c}<0.001 \\
(\mathrm{HS})\end{array}$ & $\begin{array}{c}<0.001 \\
(\mathrm{HS})\end{array}$ & $\underset{(\mathrm{NS})}{0.074}$ \\
\hline $\mathrm{HbA1C}, \mathrm{g} \%$ & $\begin{array}{c}<0.001 \\
(\mathrm{HS})\end{array}$ & $\begin{array}{c}<0.001 \\
(\mathrm{HS})\end{array}$ & $\begin{array}{l}<0.001 \\
(\mathrm{HS})\end{array}$ & $\begin{array}{l}<0.001 \\
(\mathrm{HS})\end{array}$ & $\begin{array}{c}<0.001 \\
(\mathrm{HS})\end{array}$ & $\begin{array}{c}0.089 \\
\text { (NS) }\end{array}$ \\
\hline Insulin, IU/mL & $\begin{array}{c}<0.001 \\
(\mathrm{HS})\end{array}$ & $\begin{array}{c}<0.001 \\
(\mathrm{HS})\end{array}$ & $\begin{array}{l}<0.001 \\
(\mathrm{HS})\end{array}$ & $\begin{array}{c}0.007 \\
(\mathrm{~S})\end{array}$ & $\begin{array}{c}0.019 \\
(\mathrm{~S})\end{array}$ & $\begin{array}{c}<0.001 \\
(\mathrm{HS})\end{array}$ \\
\hline HOMA-IR & $\begin{array}{c}0.147 \\
(\mathrm{NS})\end{array}$ & $\begin{array}{c}<0.001 \\
(\mathrm{HS})\end{array}$ & $\begin{array}{c}<0.001 \\
(\mathrm{HS})\end{array}$ & $\begin{array}{l}<0.001 \\
(\mathrm{HS})\end{array}$ & $\begin{array}{c}<0.001 \\
(\mathrm{HS})\end{array}$ & $\begin{array}{c}<0.001 \\
(\mathrm{HS})\end{array}$ \\
\hline
\end{tabular}

HS: Highly Significant, S: Significant, NS: Non Significant.

Table (4): Serum Fetuin-A level among studied groups.

\begin{tabular}{|c|c|c|c|c|c|c|}
\hline & \multicolumn{4}{|c|}{ Group } & \multirow[b]{2}{*}{$\begin{array}{c}\text { Total } \\
\mathbf{N}=115\end{array}$} & \multirow[b]{2}{*}{$\mathbf{p}$} \\
\hline & $\begin{array}{l}\mathrm{HCV} \\
\mathrm{N}=29\end{array}$ & $\begin{array}{c}\text { HCV\&DM } \\
\quad \mathrm{N}=29\end{array}$ & $\begin{array}{c}\mathrm{DM} \\
\mathrm{N}=29\end{array}$ & $\begin{array}{c}\text { Control } \\
\mathbf{N}=\mathbf{2 8}\end{array}$ & & \\
\hline $\begin{array}{l}\text { Fetuin-A, mg/mL } \\
(\text { Mean } \pm \text { SD) }\end{array}$ & $3.8 \pm 0.9$ & $5.5 \pm 1.5$ & $2.4 \pm 0.7$ & $1 \pm 0.3$ & $3.2 \pm 1.9$ & $\begin{array}{l}<001 \\
(\mathrm{H} \mathrm{S})\end{array}$ \\
\hline
\end{tabular}

HS: Highly Significant, S: Significant, NS: Non Significant.

N.B: Normal level of Fetuin-A 0.33 to $1.0 \mathrm{mg} / \mathrm{ml}$.

Table (5): Serum Fetuin-A level among studied groups(LSD Post-hoc test).

\begin{tabular}{|l|c|c|c|c|c|c|}
\hline & $\begin{array}{c}\text { HCV\&DM } \\
\text { Vs. DM }\end{array}$ & $\begin{array}{c}\text { HCV\&DM } \\
\text { Vs. Control }\end{array}$ & $\begin{array}{c}\text { DM Vs. } \\
\text { Control }\end{array}$ & $\begin{array}{c}\text { HCV\&DM } \\
\text { Vs. HCV }\end{array}$ & $\begin{array}{c}\text { DM Vs. } \\
\text { HCV }\end{array}$ & $\begin{array}{c}\text { HCV vs. } \\
\text { Control }\end{array}$ \\
\hline $\begin{array}{l}\text { Fetuin-A, } \\
\mathrm{mg} / \mathrm{mL}\end{array}$ & $\begin{array}{c}<0.001 \\
(\mathrm{HS})\end{array}$ & $\begin{array}{c}<0.001 \\
(\mathrm{HS})\end{array}$ & $\begin{array}{c}<0.001 \\
(\mathrm{HS})\end{array}$ & $\begin{array}{c}<0.001 \\
(\mathrm{HS})\end{array}$ & $\begin{array}{c}<0.001 \\
(\mathrm{HS})\end{array}$ & $\begin{array}{c}<0.001 \\
(\mathrm{HS})\end{array}$ \\
\hline
\end{tabular}

HS: Highly Significant, S: Significant, NS:Non Significant.

Table (6): Serum Fetuin-A level as regard HCV infection, DM and Sex.

\begin{tabular}{|c|c|c|c|c|c|c|c|c|c|}
\hline & \multicolumn{2}{|c|}{ HCV } & \multirow[b]{2}{*}{$\mathbf{p}$} & \multicolumn{2}{|c|}{ DM } & \multirow[b]{2}{*}{$\mathbf{p}$} & \multicolumn{2}{|c|}{ Sex } & \multirow[b]{2}{*}{$\mathbf{p}$} \\
\hline & $\begin{array}{c}\mathrm{HCV} \\
+\mathrm{ve} \\
\mathrm{N}=58\end{array}$ & $\begin{array}{c}\mathrm{HCV} \\
-\mathrm{ve} \\
\mathrm{N}=57\end{array}$ & & $\begin{array}{c}\mathrm{DM} \\
\mathrm{N}=58\end{array}$ & $\begin{array}{c}\text { Non- } \\
\text { DM } \\
\text { N=57 }\end{array}$ & & $\begin{array}{l}\text { Male } \\
\text { N=57 }\end{array}$ & $\begin{array}{c}\text { Female } \\
\mathbf{N}=58\end{array}$ & \\
\hline $\begin{array}{l}\text { Fetuin-A, } \\
\mathrm{mg} / \mathrm{mL} \\
(\mathrm{Mean} \pm \mathrm{SD})\end{array}$ & $\begin{array}{c}4.7 \pm \\
1.5\end{array}$ & $\begin{array}{c}1.7 \pm \\
0.9\end{array}$ & $\begin{array}{l}<0.001 \\
(\mathrm{H} \mathrm{S})\end{array}$ & $4 \pm 2$ & $\begin{array}{c}2.5 \pm \\
1.6\end{array}$ & $\begin{array}{l}<0.001 \\
(\mathrm{H} \mathrm{S})\end{array}$ & $\begin{array}{c}3.2 \pm \\
1.8\end{array}$ & $\begin{array}{c}3.2 \pm \\
2\end{array}$ & $\begin{array}{l}0.837 \\
\text { (N S) }\end{array}$ \\
\hline
\end{tabular}

HS: Highly Significant, S: Significant, NS: Non Significant. 
Table (7): Correlations between serum Fetuin-A level and the studied parameters in different studied groups

\begin{tabular}{|c|c|c|c|c|c|c|c|c|}
\hline \multirow[t]{2}{*}{ Fetuin-A, mg/mL } & \multicolumn{2}{|c|}{ HCV group } & \multicolumn{2}{|c|}{ DM group } & \multicolumn{2}{|c|}{$\begin{array}{l}\text { HCV \& DM } \\
\text { group } \\
\end{array}$} & \multicolumn{2}{|c|}{ Control group } \\
\hline & $\mathbf{r}$ & $\mathbf{p}$ & $\mathbf{r}$ & $\mathbf{p}$ & $\mathbf{r}$ & $\mathbf{p}$ & $\mathbf{r}$ & $\mathbf{p}$ \\
\hline Age, years & -0.37 & $\begin{array}{l}\mathbf{0 . 0 4 8} \\
(\mathrm{S})\end{array}$ & -0.145 & $\begin{array}{c}0.451 \\
(\mathrm{NS})\end{array}$ & -0.321 & $\begin{array}{l}0.09 \\
(\mathrm{NS})\end{array}$ & 0.004 & $\begin{array}{l}0.985 \\
(\mathrm{NS})\end{array}$ \\
\hline BMI, Kg/m2 & 0.029 & $\begin{array}{c}0.883 \\
(\mathrm{NS}) \\
\end{array}$ & 0.324 & $\begin{array}{c}0.086 \\
(\mathrm{NS}) \\
\end{array}$ & 0.101 & $\begin{array}{c}0.602 \\
(\mathrm{NS}) \\
\end{array}$ & 0.013 & $\begin{array}{c}0.948 \\
\text { (NS) } \\
\end{array}$ \\
\hline FBS, mg/dL & 0.516 & $\begin{array}{c}<0.001 \\
(\mathrm{HS}) \\
\end{array}$ & 0.245 & $\begin{array}{l}0.201 \\
(\mathrm{NS}) \\
\end{array}$ & 0.902 & $\begin{array}{c}\mathbf{0 . 0 0 1} \\
(\mathrm{HS}) \\
\end{array}$ & 0.778 & $\begin{array}{c}\mathbf{0 . 0 0 1} \\
(\mathrm{HS}) \\
\end{array}$ \\
\hline $\begin{array}{c}\text { 2-hour } \\
\text { postprandial bl. } \\
\text { glucose, } \mathrm{mg} / \mathrm{dl}\end{array}$ & 0.419 & $\begin{array}{c}<0.001 \\
(\mathrm{HS})\end{array}$ & 0.236 & $\begin{array}{l}0.235 \\
(\mathrm{NS})\end{array}$ & 0.916 & $\begin{array}{c}<\mathbf{0 . 0 0 1} \\
(\mathrm{HS})\end{array}$ & 0.758 & $\begin{array}{c}<0.001 \\
(\mathrm{HS})\end{array}$ \\
\hline HbA1C, g\% & 0.756 & $\begin{array}{c}<0.001 \\
(\mathrm{HS})\end{array}$ & 0.174 & $\begin{array}{c}0.367 \\
\text { (NS) }\end{array}$ & 0.913 & $\begin{array}{c}<0.001 \\
(\mathrm{HS})\end{array}$ & 0.053 & $\begin{array}{l}0.79 \\
\text { (NS) }\end{array}$ \\
\hline Insulin, IU/mL & 0.941 & $\begin{array}{c}<0.001 \\
(\mathrm{HS}) \\
\end{array}$ & 0.48 & $\begin{array}{c}\mathbf{0 . 0 0 8} \\
(\mathrm{S}) \\
\end{array}$ & 0.748 & $\begin{array}{l}<\mathbf{0 . 0 0 1} \\
(\mathrm{HS}) \\
\end{array}$ & 0.303 & $\begin{array}{c}0.116 \\
(\mathrm{NS}) \\
\end{array}$ \\
\hline HOMA-IR & 0.928 & $\begin{array}{c}<0.001 \\
(\mathrm{HS})\end{array}$ & 0.327 & $\begin{array}{c}0.083 \\
(\mathrm{NS})\end{array}$ & 0.913 & $\begin{array}{c}<0.001 \\
(\mathrm{HS})\end{array}$ & 0.706 & $\begin{array}{c}<0.001 \\
\text { (HS) }\end{array}$ \\
\hline AST, IU/L & -0.248 & $\begin{array}{l}0.195 \\
(\mathrm{NS}) \\
\end{array}$ & -0.036 & $\begin{array}{c}0.852 \\
\text { (NS) }\end{array}$ & -0.115 & $\begin{array}{c}0.553 \\
(\mathrm{NS}) \\
\end{array}$ & -0.072 & $\begin{array}{l}0.715 \\
\text { (NS) } \\
\end{array}$ \\
\hline ALT, IU/L & -0.346 & $\begin{array}{l}0.066 \\
\text { (NS) }\end{array}$ & -0.006 & $\begin{array}{c}0.977 \\
(\mathrm{NS})\end{array}$ & -0.066 & $\begin{array}{c}0.734 \\
(\mathrm{NS})\end{array}$ & -0.072 & $\begin{array}{c}0.716 \\
(\mathrm{NS})\end{array}$ \\
\hline TC, mg/dL & -0.119 & $\begin{array}{l}0.54 \\
(\mathrm{NS})\end{array}$ & -0.243 & $\begin{array}{l}0.204 \\
(\mathrm{NS})\end{array}$ & 0.2 & $\begin{array}{l}0.297 \\
(\mathrm{NS})\end{array}$ & -0.015 & $\begin{array}{l}0.94 \\
(\mathrm{NS})\end{array}$ \\
\hline TG, mg/dL & -0.022 & $\begin{array}{c}0.909 \\
\text { (NS) }\end{array}$ & 0.143 & $\begin{array}{c}0.459 \\
\text { (NS) } \\
\end{array}$ & -0.046 & $\begin{array}{c}0.811 \\
\text { (NS) } \\
\end{array}$ & 0.235 & $\begin{array}{c}0.229 \\
\text { (NS) } \\
\end{array}$ \\
\hline HDL, mg/dL & 0.335 & $\begin{array}{l}0.076 \\
\text { (NS) }\end{array}$ & 0.105 & $\begin{array}{c}0.587 \\
(\mathrm{NS})\end{array}$ & 0.043 & $\begin{array}{l}0.827 \\
(\mathrm{NS})\end{array}$ & 0.152 & $\begin{array}{l}0.44 \\
(\mathrm{NS})\end{array}$ \\
\hline LDL, mg/dL & 0.172 & $\begin{array}{c}0.372 \\
\text { (NS) }\end{array}$ & -0.064 & $\begin{array}{l}0.741 \\
(\mathrm{NS})\end{array}$ & 0.149 & $\begin{array}{c}0.441 \\
(\mathrm{NS})\end{array}$ & 0.144 & $\begin{array}{c}0.465 \\
\text { (NS) }\end{array}$ \\
\hline
\end{tabular}

HS: Highly Significant, S: Significant, NS: Non Significant.

$\mathrm{P} \leq 0.05=$ significant $\mathrm{p}<0.001$ highly significant and $\mathrm{p}>0.05$ Non-significant

Table (8): The validity of serum Fetuin-A as a predictor for IR in HCV patients

\begin{tabular}{|c|c|c|c|c|c|c|c|c|}
\hline $\begin{array}{c}\text { Cut-off value } \\
\text { Fetuin-A }\end{array}$ & Sensitivity & $\mathbf{9 5 \%}$ CI & Specificity & $\mathbf{9 5 \%}$ CI & +PV & $\mathbf{9 5 \%}$ CI & -PV & 95\% CI \\
\hline$>5.2 \mathrm{mg} / \mathrm{ml}$ & 54.84 & $36.0-$ & 96.30 & $81.0-$ & 94.4 & $\begin{array}{c}72.7- \\
99.9\end{array}$ & 65.0 & $\begin{array}{c}48.3- \\
79.4\end{array}$ \\
\hline
\end{tabular}




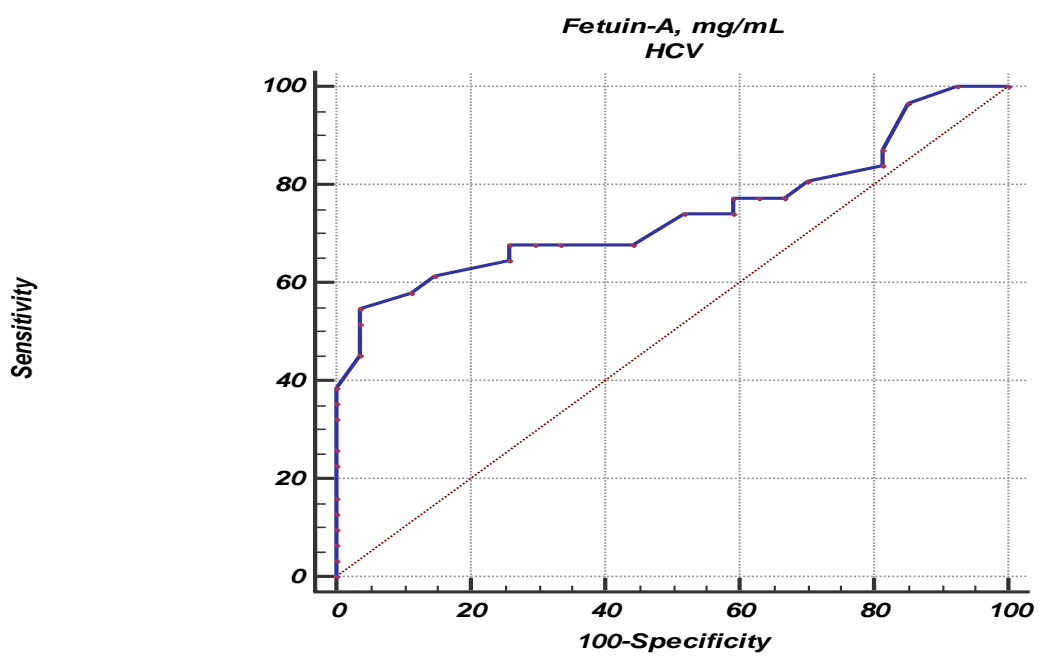

Figure (1): ROC curve of serum Fetuin-A as a predictor for IR in HCV patients.

Table (9): The validity of serum Fetuin-A as a predictor for DM in HCV patients.

\begin{tabular}{|c|c|c|c|c|c|c|c|c|}
\hline $\begin{array}{c}\text { Cut-off value } \\
\text { Fetuin-A }\end{array}$ & Sensitivity & $\mathbf{9 5 \%}$ CI & Specificity & $\mathbf{9 5 \%}$ CI & +PV & $\mathbf{9 5 \%}$ CI & -PV & 95\% CI \\
\hline$>4.9 \mathrm{mg} / \mathrm{mL}$ & 72.41 & $52.8-$ & 79.31 & $60.3-$ & 77.8 & $\begin{array}{c}57.7- \\
\text { 92 }\end{array}$ & 74.2 & $\begin{array}{c}55.4- \\
88.1\end{array}$ \\
\hline
\end{tabular}

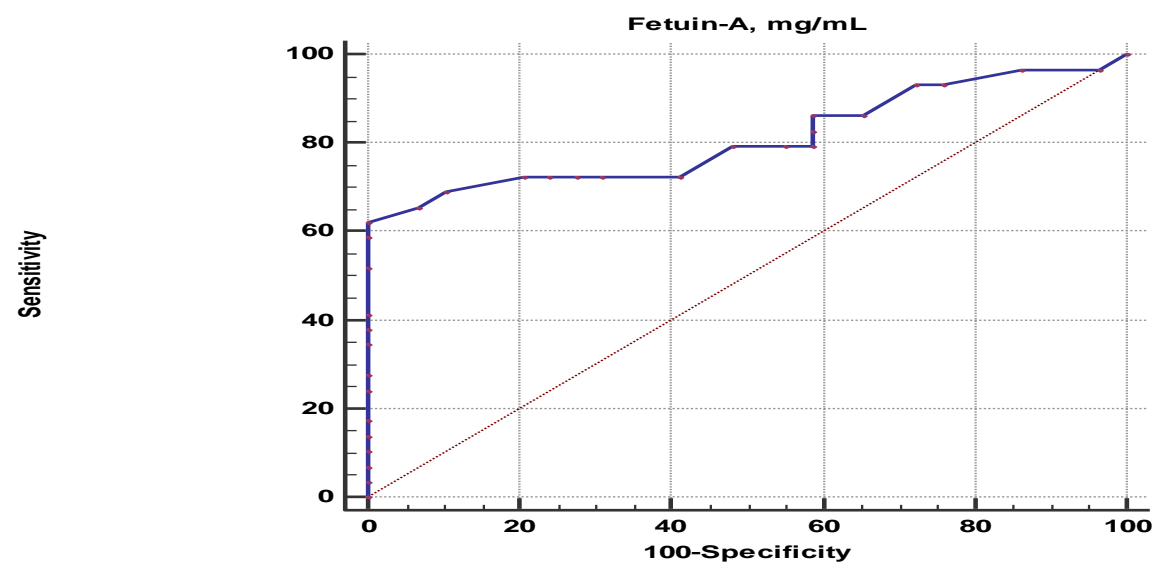

Figure (2): ROC curve of serum Fetuin-A as a predictor for DM in HCV patients.

\section{DISCUSSION}

Hepatitis $\mathrm{C}$ virus (HCV) infection is a global health problem that affects more than 170 million people worldwide. Egypt has the highest HCV prevalence in the world and its complications are among the leading public health challenges in Egypt [26]. It is a major cause of cirrhosis and hepatocellular carcinoma, making the virus the most common cause of liver failure and transplantation [27].

Insulin resistance is a consistent finding in patients with type 2 diabetes and present years before onset of diabetes [28]. Type 2 diabetes mellitus (T2DM) is a heterogeneous metabolic disease due to a progressive insulin secretory defect on the background of insulin resistance [29]. 
Hepatitis $\mathrm{C}$ may induce insulin resistance and the risk of type 2 diabetes mellitus is increased in persons with chronic hepatitis $\mathrm{C}$ about 3 folds than non-HCV patients [4].

Fetuin-A is a circulating plasma glycoprotein, produced in hepatocytes and secreted into serum, it was found to be a natural inhibitor of the insulin receptor tyrosine kinase [30]. Thus, Fetuin-A is thought to be involved in the pathogenesis of insulin resistance $[\mathbf{3 1}, \mathbf{3 2}]$.

Among the randomly selected 115 subjects included in this study the Mean \pm SD of age was $46.3 \pm 3.7$ years, $50.4 \%$ females, $49.6 \%$ males and the Mean \pm SD of BMI was $23.3 \pm 2 \mathrm{~kg} / \mathrm{m}^{2}$.

In this study, there was statistically significant difference of ALT, AST levels among studied groups which were higher in HCV \& DM followed by $\mathrm{HCV}$ when compared to DM and control groups. As the liver injury due to $\mathrm{HCV}$ and steatosis that is associated with IR is associated with increased level of ALT and AST in serum [33]. Also, Kim et al. [34] concluded that there was significant association between serum levels of liver enzymes and risk of type 2 diabetes.

There was statistically significant difference of glucose homeostasis parameters among studied groups, where HOMA-IR, fasting blood glucose, fasting serum insulin and 2-hour post prandial blood glucose level were higher among HCV \& DM than DM with statistically significant difference, as insulin resistance and T2DM represent unique extrahepatic metabolic complications for chronic hepatitis $\mathrm{C}$ virus infection [35].

Also, insulin and HOMA-IR levels were increased in DM than HCV and control subjects that were in agreement with Ahmed et al. [36].

There was statistically significant difference as regard lipid profile among different groups, where LDL, TG, TC levels were elevated significantly and HDL level was decreased in HCV\&DM followed by DM when compared to $\mathrm{HCV}$ and control groups. Kralj et al. [4] found that $\mathrm{HCV}$ infection can induce insulin resistance which is the key mechanism leading to hepatic steatosis and metabolic syndrome where dyslipidemia is associated with insulin resistance. In addition Samatha et al. [37] reported that, there was a significant elevation in the serum concentrations of: Total cholesterol, Triglycerides and low density lipoprotein (LDL) in type 2 diabetic patients compared to controls. While HDL levels were significantly decreased in type 2 diabetic patients when compared to controls.

Also, Smith and Lall [38] concluded that hypercholesterolemia, hypertriglyceridemia and elevated low density lipoprotein are the main lipid abnormalities found in diabetes which is risk for coronary artery disease.

In the current study, there was statistically significant increase of serum Fetuin-A level in HCV\&DM followed by HCV than DM and control groups. Moreover, there was statistically significant difference of serum Fetuin-A level among studied groups, where serum Fetuin-A level was higher in $\mathrm{HCV}$ patients than non-HCV subjects. This was in agreement with Sheriba et al. [39] who found that Fetuin-A was markedly higher in patients with chronic hepatitis $C$ than in healthy controls. This finding has been confirmed also by an in vitro study, which has shown that Fetuin-A level was 10 folds higher in the HCV infected hepatocellular Huh-7cell lines compared to naïve Huh-7 cells [40]. Furthermore, a recent study by a Japanese group has shown that Fetuin$A$ is a crucial constituent of cryoglobulin associated with chronic hepatitis C [41].

Serum Fetuin-A levels of HCV \& DM was higher than $\mathrm{HCV}$ patients with statistically significant difference. The increase of Fetuin-A in $\mathrm{HCV} /$ T2DM has come in accordance with a recent report revealed that the higher level of Fetuin-A is associated with IR and T2DM [42]. Moreover, it was found that single nucleotide polymorphism of Fetuin-A represents an independent risk factor for T2DM development. This result may indicate that serum Fetuin-A may play a pivotal role in IR of either DM or HCV. So,higher level was detected among concomitant DM\&HCV than $\mathrm{HCV}$ or DM alone [43].

Also, serum Fetuin-A level was statistically higher in diabetics than non diabetics with significant difference .This was in agreement with Muniyappa et al. [44]. Also, the association of higher FetuinA with incident diabetes has been reported[45].

These data were in contrast to Mori et al. [46], who found no difference in serum Fetuin-A level in type 2 diabetic patients with IR than in non diabetics. They explained their findings by the existence of glucose toxicity and/or protein modifications such as non enzymatic glycation that may overcome the effect of Fetuin-A on IR.

There was no statistically significant difference in serum Fetuin-A as regard sex. However, Sun 
et al. [47] found that serum Fetuin-A was elevated in women with T2DM.

There was a highly significant positive correlation between Fetuin-A and BMI among all studied subjects. This was in agreement with Küçük et al. [48] who found positive correlation between serum Fetuin-A and BMI. So, Fetuin-A may play an important role in the pathogenesis of metabolic syndrome through IR [49].

Also, serum Fetuin-A level was inversely correlated with age in $\mathrm{HCV}$ group that was in agreement with Nathan et al. [50]. As Fetuin-A, which is secreted from healthy hepatocytes is affected by chronic liver disease so, it will decrease with progression of the disease and aging [51].

In this study, there was positive correlation between serum Fetuin-A and TG, LDL and negative correlation with HDL level with significant difference in the whole studied subjects. These results came in consistent with the results of Ishibashi et al. [52], they concluded that elevated serum Fetuin-A is an independent marker for an atherogenic lipid profile. Also, Ix et al [53] reported that higher level of Fetuin-A was associated with higher triglycerides, LDLcholesterol, BMI, and insulin resistance. What is more, several single nucleotide polimorphisms (SNPs) of the Fetuin-A gene were found to be associated with insulin action, dyslipidemia and type 2 diabetes as well [52].

There was negative correlation between serum Fetuin-A and liver enzymes in $\mathrm{HCV}$ and HCV\&DM groups without significant difference. This is in agreement with Stefan et al. [31] who proved that Fetuin-A is a negative acute phase reactant that is down regulated in inflammation, infection and malignancy.

Serum Fetuin-A level was correlated with fasting insulin in DM group, this was in agreement with Emoto et al. [54] and Stefan et al. [43] who found that high levels of circulating Fetuin-A are associated with insulin resistance, suggesting that Fetuin-A may represent a mechanism involved in the pathophysiology of type II diabetes.

It was found that Fetuin-A has a significant positive correlation with FBG, fasting insulin and with HOMA-IR, the markers of insulin resistance in both HCV/T2 DM and HCV groups. This was in agreement with Ali et al. [42] who found that Fetuin-A is associated with HOMA-IR, metabolic syndrome and an increased risk for type 2 diabetes.
So, Fetuin-A may be a promising biochemical mediator that link $\mathrm{HCV}$ and insulin resistance [39].

Regarding the diagnostic validity of serum Fetuin-A as a predictor for IR in HCV patients at Cut-off value $>5.2 \mathrm{mg} / \mathrm{mL}$, AUC was 0.741 , the sensitivity was $54.84 \%$ and specificity was $96.30 \%$.This indicates that serum Fetuin-A has important and specific role for IR induced by $\mathrm{HCV}$ infection. Also, regarding the diagnostic validity of serum Fetuin-A as a predictor for DM in $\mathrm{HCV}$ patients at Cut-off value $>4.9 \mathrm{mg} / \mathrm{mL}$, AUC was 0.81 , the sensitivity was $72.41 \%$ and specificity was $79.31 \%$.

Finally, it can be concluded that serum level of Fetuin-A was higher in HCV patients than non HCV subjects and among diabetic patients than non diabetic subjects. Also, serum levels of Fetuin-A were markedly elevated among HCV \& DM patients followed by HCV patient groups when compared to DM and control groups. Serum Fetuin-A levels was positively correlated with glucose homeostasis parameters in both $\mathrm{HCV}$ and HCV\&DM patients. Fetuin-A, may be target for therapeutic modalities for the treatment of insulin resistance in HCV patients. Future studies on large scale to standardize cut off value of Fetuin-A as a predictor for IR and DM in HCV patients may be needed.

Funding: None.

Conflicts of interest: None.

Ethical approval: Approved.

\section{REFERENCES}

1. Lauer $\mathrm{G}$ and Walker B: Hepatitis $\mathrm{C}$ virus infection. N Engl J Med, 2001, (345): 41-52.

2. Agnello V and De Rosa F: Extrahepatic disease manifestations of HCV infection: some current issues. J Hepatol, 2004, (40): 341-352.

3. Lecube A, Hernandez C, Genesca J, Simo R: Glucose abnormalities in patients with hepatitis C virus infection: epidemiology and pathogenesis. Diabetes Care, 2006, 29: 1140-1149.

4. Kralj D, Virović L, Stojsavljević S, Duvnjak M, Smolić M, Čurčić I: Hepatitis C Virus, Insulin Resistance, and Steatosis. J Clin Trans Hepatol, 2016, 4(1): 66-75.

5. Mason A, Lau J, Hoang N, Qian K, Alexander $\mathrm{G}, \mathrm{Xu} \mathrm{L}$ et al.: Association of diabetes mellitus and chronic hepatitis $\mathrm{C}$ virus infection. Hepatology, 1999, 29(2): 328-333.

6. Vanni E, Abate M, Gentilcore E, Hickman I, Gambino R, Cassader $M$ et al.: Sites and mechanisms of insulin resistance in nonobese, 
nondiabetic patients with chronic hepatitis C. Hepatology, 2009, (50): 697-706.

7. Gutierrez-Buey G, Núñez-Córdoba J, LlaveroValero M, Gargallo J, Salvador J, Escalada J: Is HOMA-IR a potential screening test for nonalcoholic fatty liver disease in adults with type 2 diabetes ?. Eur J Intern Med, 2017, P: 1-5.

8. Knobler $\mathrm{H}$ and Schattner A: Association of hepatitis $\mathrm{C}$ and diabetes mellitus. Ann Intern Med, 2001, 135(2): 141 .

9. White D, Ratziu V, El-Serag H: Hepatitis C infection and risk of diabetes: asystematic review and meta-analysis. J Hepatol, 2008, (49): 831844.

10. Aytug S, Reich D, SapiroL,Bernstein D, Begum $\mathrm{N}$ : Impaired IRS-1/PI3-kinase signaling in patients with $\mathrm{HCV}$ :a mechanism for increased prevalence of type 2 diabetes. Hepatology, 2003, (38): 1384 1392.

11. El-Zayadi A and Anis $\mathrm{M}$ : Hepatitis $\mathrm{C}$ virus induced insulin esistance impairs response to antiviral therapy. World J Gastroenterol, 2012, (18): 212-224.

12. Nassif W, Amin A, Hassan Z, Abdelaziz D: Changes of serum omentin-1 levels and relationship between omentin-1and insulin resistance in chronic hepatitis C patients. EXCLI J, 2013, (12): 924-932.

13. Negro F: Mechanisms of hepatitis C virus-related insulin resistance. Clin. Res. Hepatol. Gastroenterol, 2011, (35): 358-363.

14. Adinolfi LE, Durante-Mangoni E, Zampino R, Ruggieron G: Hepatitis C virus-associated steatosis-pathogenic mechanisms and clinical implications. Aliment Pharmacol Ther, 2005, 22 (2): 52-55

15. Kawaguchi T, Yoshida T, Harada M, Hisamoto T, Nagao Y, Ide T et al.: Hepatitis $\mathrm{C}$ virus downregulates insulin receptor substrates 1 and 2 through up-regulation of suppressor of cytokine signaling 3. Am J Pathol, 2004,165(5): 1499-1508.

16. Shintani Y, Fujie H, Miyoshi H, Tsutsumi T, Tsukamoto K, Kimura $\mathrm{S}$ et al.: Hepatitis $\mathrm{C}$ virus infection and diabetes: direct involvement of the virus in the development of insulin resistance. Gastroenterol, 2004, 126(3): 840-848.

17. Stefan $\mathrm{N}$ and Häring $\mathrm{H}$ : The role of hepatokines in metabolism. Nat Rev Endocrinol, 2013, 9: 144-152.

18. Mathews S, Chellam N, Srinivas P, Cintron V, Leon M, Goustin A et al.: Alpha 2-HSG, a specific inhibitor of insulin receptor autophosphorylation, interacts with the insulin receptor. Mol Cell Endocrinol, 2000, (164): 87-98.

19. Denecke B, Graber S, Schafer C, Heiss A, Woltje $\mathrm{M}$, Jahnen-Dechent $\mathrm{W}$ : Tissue distribution and activity testing suggest a similar but not identical function of fetuin-B and fetuin-A. Biochem $J$, 2003, 376: 135-145.

20. Kettler M, Bongartz P, Westenfeld R, Wildberger J, Mahnken A, Böhm R et al.:Association of low fetuin-A (AHSG) concentrations in serum with cardiovascular mortality in patients on dialysis: a cross-sectional study. Lancet, 2003, 361(9360): 827-833.

21. Ix J, Wassel C, Kanaya A, Vittinghoff E, Johnson $\mathrm{K}$, Koster A et al.: Fetuin-A and Incident Type 2 diabetes Mellitus in Older Persons. JAMA, 2008, (300): 182-188.

22. Rockey D and Bissell D: Noninvasive measures of liver fibrosis. Hepatology, 2006, 43 (suppl 1): S113-S120.

23. World Health Organization (WHO): Definition and Diagnosis of Diabetes Mellitus and Intermediate Hyperglycemia: Report of a WHO/IDF Consultation. Geneva, Switzerland, 2006, 1-46.

24. World Health Organization (WHO): Obesity: Preventing and managing the global epidemic. World Health Organization.Geneva, Technical report series 894, ISBN, 2000, 92-(4)-120894120895 .

25. Pfützner A, Kunt T, Hohberg C, Mondok A, Pahler $\mathrm{S}$, Konrad $\mathrm{T}$ et al.: Fasting intact proinsulin is a highly specific predictor of insulin resistance in type 2 diabetes. Diabetes Care, 2004, (3): 682687.

26. Miller F and Abu-Raddad L: Evidence of intense ongoing endemic transmission of hepatitis C virus in Egypt. Proc Natl Acad Sci USA, 2010, 107(33): 14757-14762.

27. Brown R, O'Leary J, Reddy K,Kuo A, Morelli $\mathrm{G}$, Burton $\mathrm{J}$ et al.: Interferon-free therapy for genotype 1 hepatitis $\mathrm{C}$ in liver transplant recipients: Real-world experience from the hepatitis $\mathrm{C}$ therapeutic registry and research network. Liver Transpl, 2016, (22): 24-39.

28. Gutierrez-Buey G, Núñez-Córdoba J, LlaveroValero M, Gargallo J, Salvador J, Escalada J: Is HOMA-IR a potential screening test for nonalcoholic fatty liver disease in adults with type 2 diabetes?. Eur J Intern Med, 2017, P: 1-5.

29. American Diabetes Association (ADA): Standards of Medical Care in Diabetes. Diabetes Care, 2015, 38(Suppl 1): S4-89.

30. Hennige A, Staiger H, Wicke C, Machicao F, Fritsche A, Häring $\mathrm{H}$ et al.: Fetuin-A induces cytokine expression and suppresses adiponectin production. PLoS One, 2008, 3(3): e1765.

31. Stefan N, Hennige A, Steiger H, Machann J, Schick F, Kröber S et al.: Alpha2-HeremansSchmid glycoprotein/ fetuin-A is associated with insulin resistance and fat accumulation in the liver in humans. Diabetes Care, 2006, 29(4): 853-857. 
32. Tekeoğlu İ, Harman $\mathrm{H}$, Sağ S, Altındiş M, Kamanlı A, Nas K: Levels of serum pentraxin 3, IL-6, fetuin A and insulin in patients with rheumatoid arthritis. J Cyto, 2016, (83): 171-175.

33. Ruhl $\mathrm{C}$ and Everhart J: Elevated serum alanine aminotransferase and gamma-glutamyltransferase and mortality in the United States population. Gastroenterology, 2009, 136: 477-485.

34. Kim C, Park J, Lee K, Kim J, Kim K: Association of serum $\gamma$-glutamyltransferase and alanine aminotransferase activities with risk of type 2 diabetes mellitus independent of fatty liver. Diabetes/Metab Res, 2009, (25): 64-69.

35. Mangia A and Ripoli M: Insulin resistance, steatosis and hepatitisC virus. Hepatol Int, 2013, (7): 782789.

36. Ahmed L, Gomaa S, Abd El-Ghaffar N, Ahmed Z, Yousry L, Rabea M: Fetuin-A and type II diabetes mellitus. The Egyptian Journal of Internal Medicine, 2014, (26): 157-161.

37. Samatha P, Venkateswarlu M, Siva V: Lipid Profile Levels in Type 2 Diabetes Mellitus from the Tribal Population of Adilabad in Andhra Pradesh, India. J Clin Diag Res, 2012, (6): 590592.

38. Smith S and Lall A: A Study on Lipid Profile Levels of Diabetics and Non-Diabetics Among Naini Region of Allahabad, India. Turkish Journal of Biochemistry, 2008, (33):138-141.

39. Sheriba N, Sabry I, Hendawy L, Zaky M: Study of Fetuin A in patients with chronic HCV with or without Type 2 Diabetes Mellitus and its relation to insulin resistance. International Journal of Recent Scientific Research, 2016, (7):1144511447.

40. Singaravelu R, Blais D, McKay C, Pezacki J: Activity-based protein profiling of the hepatitis $\mathrm{C}$ virus replication in Huh-7 hepatoma cells using a non-directed active site probe. Proteome $S c i$, 2010, (8): 5-21.

41. Okazaki T, Yamazaki K, Iwasaki T, Okano T, Kurosaki Y, Nakamura K et al.: Alpha 2-HS glycoprotein is an essential com-ponent of cryoglobulin associated with chronic hepatitis C. Clin Chem Lab Med, 2013, (51): 17-28.

42. Ali S, Nassif W, Abdelaziz D: Alterations in serum levels of Fetuin A and Selenoprotein $\mathrm{P}$ in chronic hepatitis C Patients with concomitant Type 2 diabetes.Clin Res Hepatol Gastroenterol, 2016, 40 (4): 465-470.

43. Stefan N, Sun Q, Fritsche A, Machann J, Schick F, Gerst $F$ et al.: Impact of the adipokine adiponectin and the hepatokine Fetuin-A on the development of type 2 diabetes: prospective cohort-and cross-sectional phenotyping studies. PLoS One, 2014, (9): 1-8.
44. Muniyappa R, Lee S, Chen H, Quon M: Current approaches for assessing insulin sensitivity and resistance in vivo: advantages, limitations, and appropriate usage. Am J Physiol Endocrinol Metab, 2008, (294): 15-26.

45. Kanaya A, Harris T, Goodpaster B, Tylavsky F, Cummings S: Adipocytokines attenuate the association between visceral adiposity and diabetes in older adults. Diabetes Care, 2004, (27):1375-1380.

46. Mori K, Emoto M, Yokoyama H, Araki T, Teramura M, Koyama $\mathrm{H}$ et al.: Association of Serum Fetuin-A with Insulin Resistance in Type 2 Diabetic and Non diabetic Subjects. Diabetes Care, 2006, (29): 462-468.

47. Sun Q, Cornelis M, Manson J, Hu F: Plasma levels of fetuin-A and hepatic enzymes and risk of type 2 diabetes in women in the U.S. Diabetes, 2013, 62(1): 49-55.

48. Küçük S, Koçak H, İyidoğan Y, Seyithanoğlu M, Topparmak E, Kayan-Tapan T et al.: Serum Fetuin-A and arginase-1 in human obesity model: Is there any interaction between inflammatory status and arginine metabolism? Scandinavian Journal of Clinical \& Laboratory Investigation, 2015, (1): 7-19.

49. Ix J, Shlipak M, Brandenburg V, Ali S, Ketteler M, Whooley M: Association between human fetuin-A and the metabolic syndrome: Data from the Heart and Soul Study. Circulation, 2006, (113): 1760-1767.

50. Nathan T, Jennifer A, James M, Sarah W: Plasma Fetuin-A concentrations in young and older highand low-active men. Metabolism, 2011, 60(2): 265-271.

51. Schulze M, Schulz M, Heidemann C, Schienkiewitz A, Hoffmann K, Boeing H: Carbohydrate intake and incidence of type 2 diabetes in the European Prospective Investigation into Cancer and Nutrition (EPIC)-Potsdam Study. Br J Nutr, 2008, (99): 1107-1116.

52. Ishibashi A, Ikeda Y, Ohguro T, Kumon Y, Yamanaka S, Takata H et al.: Serum fetuin-A is an independent marker of insulin resistance in Japanese men. J Atheroscler Thromb, 2010, (17): 925-933.

53. Ix J, Biggs M, Mukamal K, Kizer J, Zieman S, Siscovick D et al.: Association of fetuin-A with incident diabetes mellitus in community-living olderadults: the cardio-vascular health study. Circulation, 2012, (125): 2316-2322.

54. Emoto M, Mori K, Lee E, Kawano N, Yamazaki Y, Tsuchikura S et al.: Fetuin-A and atherosclerotic calcified plaque in patients with type 2 diabetes mellitus. Metabolism, 2010, 59(6): 873-878. 